\title{
BMJ Open Effect of individual patient risk, centre, surgeon and anaesthetist on length of stay in hospital after cardiac surgery: Association of Cardiothoracic Anaesthesia and Critical Care (ACTACC) consecutive cases series study of 10 UK specialist centres
}

\author{
Olympia Papachristofi, ${ }^{1}$ Andrew A Klein, ${ }^{2}$ John Mackay, ${ }^{2}$ Samer Nashef, ${ }^{3}$ \\ Nick Fletcher, ${ }^{4}$ Linda D Sharples, ${ }^{1}$ on behalf of the Association of Cardiothoracic \\ Anaesthesia and Critical Care (ACTACC)
}

To cite: Papachristofi 0 , Klein AA, Mackay J, et al. Effect of individual patient risk, centre, surgeon and anaesthetist on length of stay in hospital after cardiac surgery: Association of Cardiothoracic Anaesthesia and Critical Care (ACTACC) consecutive cases series study of $10 \mathrm{UK}$ specialist centres. BMJ Open 2017;7:e016947. doi:10.1136/ bmjopen-2017-016947

- Prepublication history and additional material for this paper are available online. To view please visit the journal (http:// dx.doi.org/10.1136/bmjopen2017-016947).

Received 22 March 2017 Revised 4 July 2017 Accepted 20 July 2017

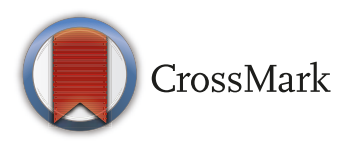

For numbered affiliations see end of article.

\section{Correspondence to} Dr Olympia Papachristofi; olympia.papachristofi@Ishtm. ac.uk

\section{ABSTRACT}

Objectives To determine the relative contributions of patient risk profile, local and individual clinical practice on length of hospital stay after cardiac surgery.

Design Ten-year audit of prospectively collected consecutive cardiac surgical cases. Case-mix adjusted outcomes were analysed in models that included random effects for centre, surgeon and anaesthetist.

Setting UK centres providing adult cardiac surgery. Participants 10 of 36 UK specialist centres agreed to provide outcomes for all major cardiac operations over 10 years. After exclusions (duplicates, cases operated by more than one consultant, deaths and procedures for which the EuroSCORE risk score for cardiac surgery is not appropriate), there were 107038 cardiac surgical procedures between April 2002 and March 2012, conducted by 127 consultant surgeons and 190 consultant anaesthetists.

Main outcome measure Length of stay (LOS) up to 3 months postoperatively.

Results The principal component of variation in outcomes was patient risk (represented by the EuroSCORE and remaining patient heterogeneity), accounting for $95.43 \%$ of the variation for postoperative LOS. The impact of the surgeon and centre was moderate (intra-class correlation coefficients ICC $=2.79 \%$ and $1.59 \%$, respectively), whereas the impact of the anaesthetist was negligible (ICC $=0.19 \%)$. Similarly, $96.05 \%$ of the variation for prolonged LOS (>11 days) was attributable to the patient, with surgeon and centre less but still influential components (ICC $=2.12 \%$ and $1.66 \%$, respectively, $0.17 \%$ only for anaesthetists). Adjustment for year of operation resulted in minor reductions in variation attributable to surgeons (ICC $=2.52 \%$ for LOS and $2.23 \%$ for prolonged LOS).

Conclusions Patient risk profile is the primary determinant of variation in LOS, and as a result, current initiatives to reduce hospital stay by modifying consultant performance are unlikely to have a substantial impact.

\section{Strengths and limitations of this study}

The study comprises more than 100000 cases from 10 of 36 UK specialist centres, amounting to almost one third of the cardiac cases in the UK between 2002 and 2012.

- The study is the first to examine the impact of the operating centre and key providers involved in the delivery of care on the length of stay (LOS) after cardiac surgery.

- Identifying how these external factors influence LOS may contribute to improving the efficiency of care. Total hospital LOS may have been underestimated due to lack of information on periods of time after inter-hospital transfer.

- The study concerned specialist centres with a likely interest in quality improvements therefore its findings may not be generalisable to smaller, nonspecialist centres.

Therefore, substantially reducing hospital stay requires shifting away from a one-size-fits-all approach to cardiac surgery, and seeking alternative treatment options personalised to high-risk patients.

\section{INTRODUCTION}

According to 2013 records, about 36000 patients undergo cardiac surgery in the UK each year at a high annual cost of around $£ 300$ million. ${ }^{1}$ Anticipated below-inflation increases in future National Health Service (NHS) tariffs have inevitably triggered the search for efficiency savings, particularly improved patient throughput accompanied 
by shorter hospital stay, which is a key driver of surgical costs. ${ }^{23}$ Besides financial benefits for the NHS, improving efficiency through length-of-stay (LOS) reductions will also yield benefits for patients as prolonged LOS is directly associated with increased risk of complications and personal financial burden. ${ }^{2-5}$ Reductions in LOS could release capacity in the system (e.g. release of occupied beds, nurse/doctor time), allowing the reallocation of limited NHS resources to other areas in need. Understanding the causes of prolonged LOS may also lead to practices that contribute to the reduction of postoperative complications and other adverse events that have a negative impact on the quality of life of patients.

Despite operating on relatively homogeneous patient populations, previous benchmarking exercises have identified considerable centre differences in postoperative models of care and LOS after cardiac surgery. ${ }^{6}$ Differences in healthcare professionals' practices may also influence hospital stay; ${ }^{7}$ nevertheless, the impact of individual surgeons and anaesthetists on LOS has received less attention. For instance, the operating surgeon has been shown to have a significant impact on in-hospital mortality post cardiac surgery. ${ }^{78}$ However, to our knowledge, their impact on postoperative LOS has not been explored. Technically skilled surgeons with low postoperative morbidity should achieve lower LOS. ${ }^{39-12}$ Similarly, previous studies have suggested differences in anaesthetic practices; for example, the use of 'fasttrack' anaesthesia protocols may accomplish a similar goal ${ }^{1314}$ however, the evidence has been inconclusive. ${ }^{15}$ These relationships may be confounded by changes in service provision over time, so that careful analysis is required.

Several authors have studied the association between patient-related factors (e.g. disease severity, existence of comorbidities) and prolonged LOS after cardiac surgery. ${ }^{16-18}$ There is also controversy as to whether different practices at different centres in the UK directly impact on hospital stay after cardiac surgery. ${ }^{19}$ This study aims to quantify the variation in risk-adjusted postoperative LOS between cardiac centres, surgeons and anaesthetists across the UK, and to investigate changes in these components over time.

\section{METHODS}

\section{Data source}

Cohorts comprising consecutive case series from UK specialist cardiac centres were provided to the Association of Cardiothoracic Anaesthesia and Critical Care. Data collection is mandated by the NHS and recorded prospectively in each centre. Requirement for formal ethical approval was waived according to the National Research Ethics Service of the NHS Health Research Authority. Previously published work on this dataset examined the impact of the anaesthetist, surgeon and centre on in-hospital mortality. ${ }^{7}$

\section{Study cohort}

Details of how the study cohort was derived have been previously published. Briefly, our cohort comprised 10 out of 36 UK specialist cardiac centres that provided datasets totalling more than 100000 cardiac surgical patients (figure 1). All 36 UK specialist cardiac centres were approached, of which 10 agreed to participate and obtained local permissions for data provision within a set time frame of a month. No centres were excluded. Data from consecutive major cardiac operations were prospectively collected for the 10-year period April 2002 to March 2012. Exclusion criteria were procedures for which the Logistic EuroSCORE (see 'Variables and outcome measures' section for detailed description) was not appropriate, cardiac transplants, pulmonary endarterectomy procedures and very high-risk cases that necessitated delivery by at least two consultant surgeons. Patients under 18 years old were also excluded $(0.08 \%)$. Patients with multiple operations at distinct admissions during the study period were treated as independent episodes.

There was a small amount of missing provider data $(\mathrm{n}=28,0.02 \%$ and $\mathrm{n}=1482,1.3 \%$ missing surgeon and anaesthetist entries, respectively) which were excluded from the analysis. A small number of cases with missing discharge destination $(n=129,0.11 \%)$ or date $(n=125$, $0.11 \%$ ) were excluded. Finally, the EuroSCORE was not recorded for 755 entries $(0.66 \%)$ which were also excluded. There were 3 patients with unknown sex, 40 with unknown operative priority status and 5964 with unrecorded operation type, all of whom were included in the analysis (table 1).

Surgeons and anaesthetists with caseloads smaller than $0.1 \%$ of the total caseload of their centre were excluded; these professionals, with the exception of one surgeon, had carried out fewer than 10 operations and had either retired just after the onset of the study period, were appointed just before the end of the study period or held short-term contracts. Patients who were not discharged after 3 months of the procedure date were excluded from the analysis as any patient-related outcomes would likely be unrelated to the procedure itself, and more likely be a result of other comorbidities $(\mathrm{n}=272,0.24 \%)$. Moreover, all cases with immediate discharge (i.e. zero LOS) were excluded as they were either deaths or transfers to other centres $(n=441,0.4 \%)$. All remaining cases that resulted in in-hospital death were also excluded from the analysis in order to avoid bias associated with short LOS due to early death considered as a positive outcome and to be consistent with published literature $(\mathrm{n}=2971,2.7 \%){ }^{16}{ }^{18}$

The final analysis dataset comprised 107038 cases (93\% of the original case series, $\mathrm{n}=115254$ ) treated by 127 surgeons and 190 anaesthetists in 10 centres. The dataset comprised $91 \%(\mathrm{n}=127$ of 140$)$ and $76 \%(\mathrm{n}=190$ of 250) of the initial surgeon and anaesthetist samples, respectively; providers were excluded principally due to low caseload volumes. 


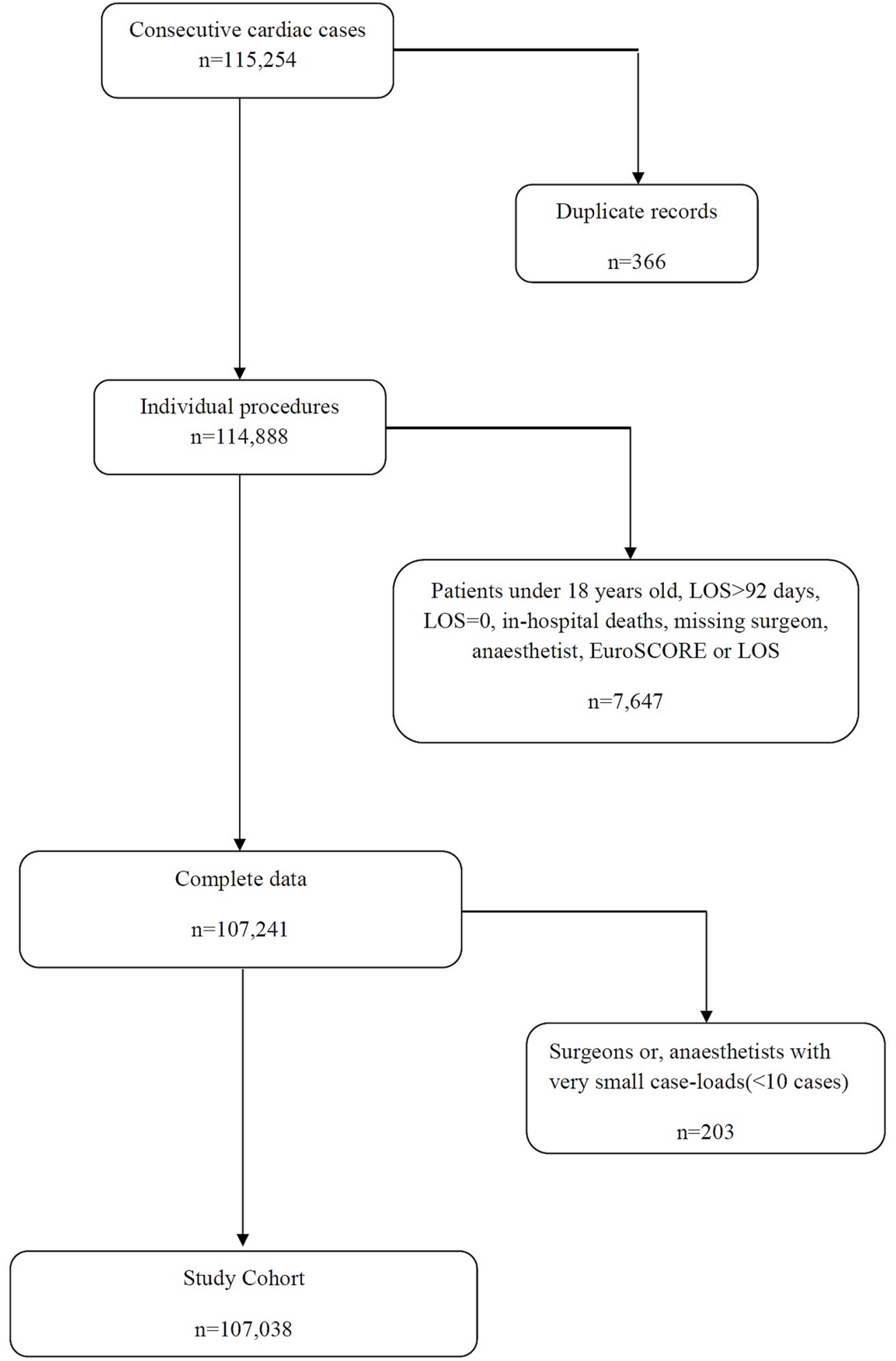

Figure 1 Flow diagram showing how the final dataset was derived. LOS, length of stay.

\section{Patient involvement}

No patients were involved in setting the research question or the outcome measures, developing plans for design or implementation of the study. No patients were asked to advise on interpretation and writing up of results. There are no plans to disseminate the results of the research to study participants or the relevant patient community.

\section{Variables and outcome measures}

The primary outcome measure was LOS up to 3 months postoperatively. LOS was defined as the number of days spent in hospital from the day of surgery to hospital discharge. The secondary measure of interest was prolonged LOS, defined as a hospitalisation of more than 11 days following surgery. There is no consensus in the 
Table 1 Patient and operative characteristics for the analysis dataset $(n=107038)$

\begin{tabular}{|c|c|c|}
\hline Characteristics & Category & $\begin{array}{l}\text { Frequency } \\
\text { (percentage) }\end{array}$ \\
\hline \multicolumn{3}{|l|}{$\begin{array}{l}\text { Patient } \\
\text { characteristics }\end{array}$} \\
\hline \multirow{2}{*}{$\begin{array}{l}\text { Age at admission } \\
\text { (years) }\end{array}$} & $18-36$ & $1883(1.76)$ \\
\hline & $36-56$ & $15149(14.15)$ \\
\hline $\begin{array}{l}{ }^{*} \text { Mean: } 66.20 \\
(11.31)\end{array}$ & $56-66$ & $28502(26.63)$ \\
\hline Median: 68 & $66-76$ & $39720(37.11)$ \\
\hline \multirow[t]{2}{*}{ IQR: 60-74 } & $76-86$ & 20682 (19.32) \\
\hline & $86-96$ & $1102(1.03)$ \\
\hline \multirow[t]{3}{*}{ Gender } & Male & 78261 (73.12) \\
\hline & Female & $28774(26.88)$ \\
\hline & Unknown & $3(<0.01)$ \\
\hline $\begin{array}{l}\text { EuroSCORE } \\
\text { (probability) }\end{array}$ & $0-0.1$ & 87559 (81.80) \\
\hline $\begin{array}{l}{ }^{*} \text { Mean: } 0.0690 \\
(0.0896)\end{array}$ & $0.1-0.2$ & 12515 (11.69) \\
\hline Median: 0.0400 & $0.2-0.3$ & $3693(3.45)$ \\
\hline $\begin{array}{l}\text { IQR: } 0.0208- \\
0.0777\end{array}$ & $\geq 0.3$ & 3271 (3.06) \\
\hline
\end{tabular}

\begin{tabular}{llc}
$\begin{array}{l}\text { Operative } \\
\text { characteristics }\end{array}$ & & \\
Priority & Elective & $74909(69.98)$ \\
& Urgent & $28312(26.45)$ \\
& Emergency & $3525(3.30)$ \\
& Salvage & $252(0.23)$ \\
\hline & Unknown & $40(0.04)$ \\
\hline Operation type & CABG (isolated) & $56586(52.87)$ \\
& AVR (isolated) & $9719(9.08)$ \\
& MVR+other & $6178(5.77)$ \\
& CABG+AVR & $8594(8.03)$ \\
& CABG+other & $2204(2.06)$ \\
& procedures & \\
& CABG+other valve & $2860(2.67)$ \\
& Other procedures & $3800(3.55)$ \\
\hline & AVR+other & $2511(2.34)$ \\
& procedures & \\
& $\begin{array}{l}\text { CABG+AVR+other } \\
\text { procedures }\end{array}$ & $1292(1.21)$ \\
& Valve alone & $5788(5.41)$ \\
\hline & Valve+other & $1542(1.44)$ \\
procedures & \\
Unknown & $5964(5.57)$ \\
\hline
\end{tabular}

*For continuous variables, the mean (SD), median and IQR are shown and their intervals are given under the column 'Category'. AVR, aortic valve replacement or repair; CABG, coronary artery bypass grafting; MVR, mitral valve replacement or repair. literature on the definition of prolonged LOS after cardiac surgery, and as a result, published studies often adopt the 75 th centile of the LOS distribution. ${ }^{16} 172021$ In our data set this corresponded to 11 days, and we have chosen it as the cut-off for prolonged stay to ensure consistency with published literature; we sought the expert advice of our cardiac surgical collaborators to ensure this was relevant to cardiac surgery in the NHS setting.

Since there is no established risk score for prolonged LOS, adjustment for varying patient case-mix risk was achieved using the logistic EuroSCORE. ${ }^{22}$ The logistic EuroSCORE is a very well-established risk score for in-hospital death postcardiac surgery with widespread use worldwide and involves 17 cardiac, operation-related and patient-related factors. The recently recalibrated version of the score (EuroSCORE II) was not available at the study onse ${ }^{23}$; our analysis included the original logistic EuroSCORE as this was the one used by the participating centres. One centre used the additive EuroSCORE which is associated with underprediction in high-risk cases. The proportion of high-risk patients for which the additive EuroSCORE is known to underperform (additive EuroSCORE $\geq 10 \%$ ) was very small $(0.5 \%, \mathrm{n}=586 \text { of } 107038)^{24}$, and results of sensitivity analysis excluding this centre did not differ from analysis of the full cohort. We considered using patient age, sex and urgency instead of the logistic EuroSCORE to account for patient heterogeneity, but EuroSCORE provided better model fit to the data, based on statistical criteria. In addition to variation due to centre, surgeon and anaesthetist, the covariate of interest was the calendar year of operation.

\section{Statistical methods}

We investigated the relationship between LOS up to 3 months postoperatively and potential covariates using mixed effects regression models. Patients were clustered within surgeons and anaesthetists who in turn, were clustered within centres inducing a hierarchy. To reflect this, random effects terms were included for centres, surgeons and anaesthetists. Logistic EuroSCORE was included as a fixed effect in all models to adjust for varying patient case-mix risk; year of procedure was included as a continuous fixed effect to investigate changes in outcomes over time.

Since the primary LOS outcome was positively skewed, linear mixed effects models were fitted to the logarithm of the LOS $(\log (\operatorname{LOS}))$. Prolonged LOS was modelled as a binary endpoint ( $\leq 11 \mathrm{vs}>11$ days) using logistic mixed effects models. The following models were implemented for both outcomes of interest.

Initially, two three-level random intercept models were fitted in order to establish individual surgeon and anaesthetist effects on the patient outcome, controlling for centre effects and patient case-mix risk. Thereafter, in order to model the effects of surgeons and anaesthetists simultaneously, we fitted a three-level cross-classified model assuming an additive 
contribution (on the log scale) from each provider (anaesthetist and surgeon), clustered within centres. We further fitted a two-level centre random intercept model, accounting solely for patient heterogeneity, in order to compare its outputs to those of the threelevel cross-classified model and assess the impact of provider adjustment on between-centre variation. In order to investigate the effect of time, we included the year of operation in the three-level cross-classified model. The methodology used has been described in detail in Papachristofi et al. ${ }^{25}$

Finally, in each model we estimated the intra-class correlation coefficients (ICCs) ${ }^{26}$ which represent the proportion of the total variation in the outcome that is attributable to each of the anaesthetist, surgeon and centre. The likelihood ratio test was used to determine the significance of the fixed effects terms and the relevant $p$ values. We implemented all our methods using the statistical software $\mathrm{R}(\mathrm{V} .3 .2 .2) .{ }^{27} 28$

\section{RESULTS}

Baseline characteristics for the study cohort are summarised in table 1. Almost three-quarters of the patients were men $(73.1 \%)$. The mean (SD) age of our cohort was 66.20 (11.31) years. Overall, the median postoperative LOS over the 10 -year study period was 7 days, with $75 \%$ of patients discharged between 6 and 11 days; the corresponding mean LOS was 10.19 (8.36) days, although this is influenced by a small proportion of large values. The mean LOS over time in each centre is depicted in figure 2, which shows varying patterns across centres; for instance, LOS decreased over time in centre 6 whereas it increased in centre 8. Summaries of each centre's cohorts are given in table 2. Almost $23 \%$ of the study cohort had prolonged LOS over 11 days, which was associated with higher operative risk score compared to patients with LOS of 11 days or less (mean EuroSCORE 11.28\% vs 5.42\%); a histogram of the distribution of surgeon caseload volume is provided in the online supplementary figure S1.

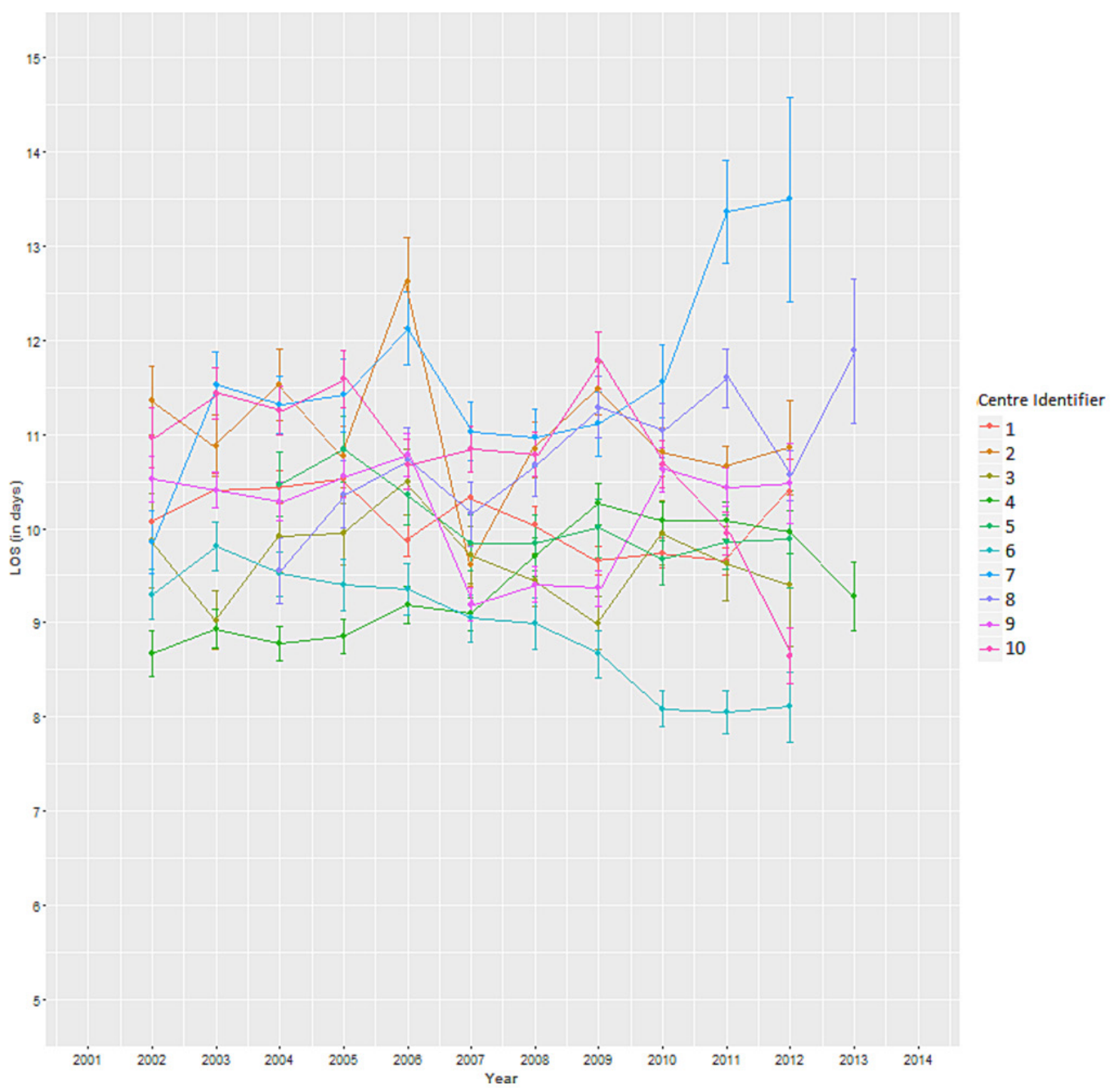

Figure 2 Mean postoperative length of stay (LOS) in hospital and 95\% Cls over time for each participating centre. 
Table 2 Number of patients operated on, surgeons and anaesthetists in each centre during the study period.

\begin{tabular}{lccclcl}
\hline Centre no & Patients & Surgeons & Anaesthetists & $\begin{array}{l}\text { LOS } \\
\text { median (IQR) }\end{array}$ & \multicolumn{1}{l}{$\begin{array}{l}\text { LOS } \\
\text { mean (SD) }\end{array}$} & Logistic EuroSCORE \\
\hline 1 & 17889 & 21 & 24 & $8(6-11)$ & $10.06(7.14)$ & $7.52(9.74) \%$ \\
\hline 2 & 9323 & 13 & 16 & $8(6-12)$ & $10.96(9.13)$ & $8.92(11.26) \%$ \\
3 & 6357 & 6 & 8 & $7(6-10)$ & $9.69(8.59)$ & $7.62(9.03) \%$ \\
4 & 15008 & 16 & 24 & $7(6-10)$ & $9.47(7.47)$ & $5.77(7.26) \%$ \\
\hline 5 & 6661 & 10 & 15 & $7(6-11)$ & $10.08(8.79)$ & $6.13(7.96) \%$ \\
$6^{*}$ & 9637 & 10 & 17 & $7(6-9)$ & $9.03(7.76)$ & $4.29(3.18) \%$ \\
\hline 7 & 7537 & 13 & 17 & $8(6-13)$ & $11.41(9.86)$ & $7.48(10.61) \%$ \\
8 & 7238 & 11 & 13 & $7(6-11)$ & $10.75(9.08)$ & $6.71(9.90) \%$ \\
\hline 9 & 16506 & 17 & 22 & $7(6-11)$ & $10.15(8.47)$ & $7.47(9.58) \%$ \\
\hline 10 & 10882 & 10 & 34 & $8(8-12)$ & $10.95(8.75)$ & $6.91(7.84) \%$ \\
\hline
\end{tabular}

Surgeons and anaesthetists who looked after $<10$ patients were excluded. Values are frequency or mean (SD) unless specified as median (IQR)

${ }^{*}$ Additive EuroSCORE was provided by this centre. LOS, length of stay.

The logistic EuroSCORE was significantly associated with LOS in both surgeon and anaesthetist models, additionally adjusted for centre effects (1.230, 95\% CI 1.226 to 1.234 and $1.229,95 \%$ CI 1.225 to 1.232 , respectively, $\mathrm{p}$ value $<0.0001$ for both). This amounted to an increase in LOS of about $23 \%$ for each $1 \%$ increase in logistic EuroSCORE. The logistic EuroSCORE remained significant in the three-level cross-classified model including both surgeon and anaesthetist effects $(1.231,95 \%$ CI 1.226 to 1.234 , p value $<0.0001$ ). Table 3 shows that $95.43 \%$ of the variation in $\log (\mathrm{LOS})$ in this analysis was attributable to the EuroSCORE (and remaining patient heterogeneity).

Figure 3A, B shows the estimated LOS, in days, with its 95\% CI for each surgeon for a patient of average risk (i.e. mean EuroSCORE estimated at $6.9 \%$ ), adjusting solely for centre effects and adjusting for centre and anaesthetist effects simultaneously. Estimated LOS for 18 out of 127 surgeons, from nine different centres, have 95\% CI lying wholly below the average LOS, suggesting shorter hospitalisations for their caseload. Fifteen surgeons from seven centres had higher than average estimated LOS. The surgeon random effects variance was modest yet important, with $\mathrm{ICC}_{\text {surgeon }}=0.0287$ suggesting $2.87 \%$ of the variation in outcome is attributable to the operating surgeon. Adjusting for anaesthetist effects resulted in a minor decrease in the $\mathrm{ICC}_{\text {surgeon }}$ from 0.0287 to 0.0279 .

Table 3 Percentage of the variation in postoperative length of stay (LOS) and prolonged LOS attributed to each component

Patient and other

Outcome Centre Surgeon Anaesthetist covariates

$\begin{array}{lllll}\text { LOS } & 1.59 & 2.79 & 0.19 & 95.43 \\ \begin{array}{l}\text { Prolonged } \\ \text { LOS }\end{array} & 1.66 & 2.12 & 0.17 & 96.05\end{array}$

The surgeons with longest and shortest average LOS were distributed across seven centres, hence we could not identify a specific centre of extreme performance. This finding, in conjunction with the $\operatorname{ICC}_{\text {centre }}(1.59 \%)$, suggests that LOS is influenced by both surgeon and, to a small extent, by the operating centre.

Figure 3C, D depicts the analogous anaesthetist forest plots, controlling solely for centre effects, and controlling for centre and surgeon effects simultaneously. Between-anaesthetist variability in LOS is smaller than between-surgeon variability (figure 3C), with associated $\mathrm{ICC}_{\text {anaesthetist }}$ of $0.58 \%$. Estimated LOS durations for 10 out of 190 anaesthetists, from five different centres, have $95 \%$ CI lying wholly below the average LOS indicating better performance that average. There were 14 anaesthetists from nine centres whose estimated LOS was higher than average. However, once surgeon effects were adjusted for, anaesthetist variation reduced to ICC $_{\text {anaesthe- }}$ tist $=0.0019(0.19 \%)$, which is negligible. Figure 3D indicates that there is only one remaining anaesthetist with 95\% CI wholly below the average; likewise, the number of anaesthetists with estimated LOS above the average reduced from 14 to 4 , employed in four different centres. This is unsurprising as, by pure chance, we would expect approximately five anaesthetists to lie at the upper end of the spectrum (i.e. if anaesthetists were normally distributed, $2.5 \%$ of $190(\mathrm{n}=4.75)$ would lie above the $97.5 \%$ quantile). The difference in estimated LOS between the two anaesthetists at the extremes reduced from almost $2 \frac{1}{2}$ days to less than 1 day.

Adjusting only for patient heterogeneity, the proportion of variation attributed to centre where the procedure was undertaken was $1.79 \%\left(\mathrm{ICC}_{\text {centre }}=0.0179\right)$. When surgeon and anaesthetist effects were added, ICC centre $_{\text {en }}$ reduced to $1.59 \%$; comparison of figure $4 \mathrm{~A}$, B indicates that two centres remained significantly above, and two below, the overall average. 


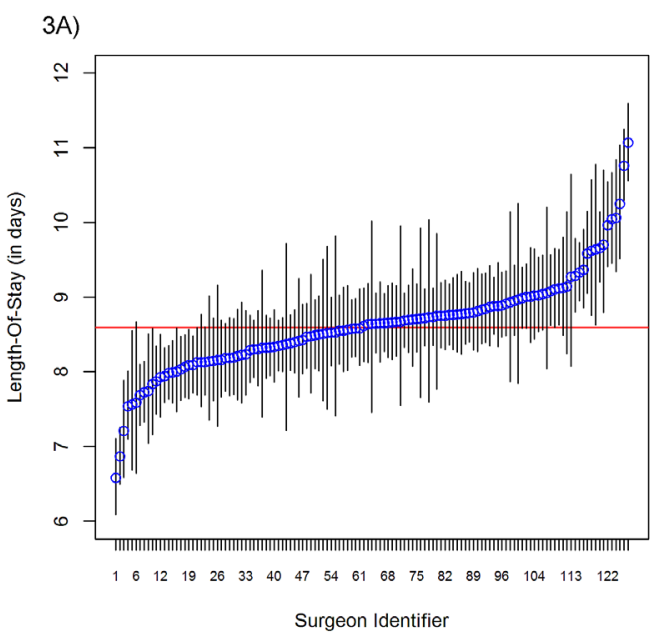

3B)

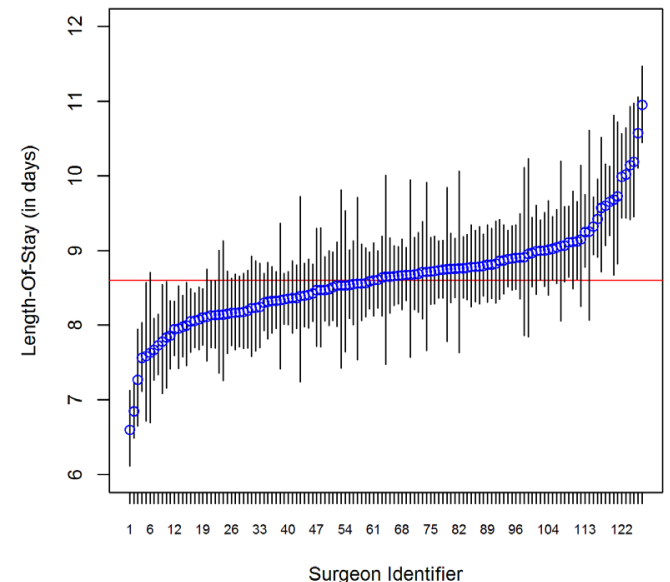

3C)

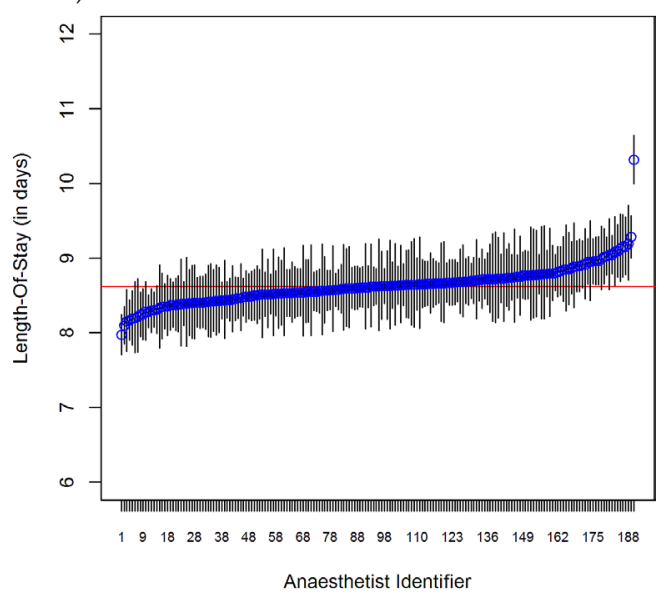

3D)

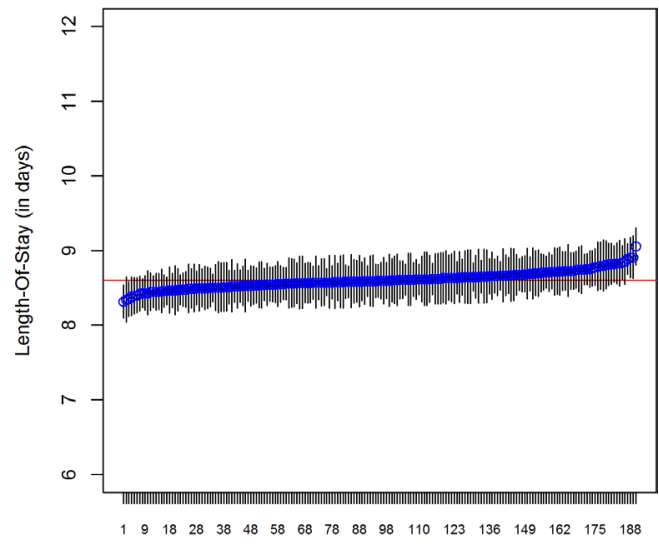

Anaesthetist Identifier

Figure 3 Estimated mean postoperative length of stay (LOS) in hospital and 95\% Cl for each surgeon (A, B) and anaesthetist $(C, D)$ for a patient with average EuroSCORE risk. Horizontal line is the estimated average LOS for a patient with average EuroSCORE.

The effect of calendar year of operation on LOS was statistically significant $(0.994,95 \%$ CI 0.993 to $0.995, \mathrm{p}$ value $<0.0001)$. However, this amounted to a decrease of $0.6 \%$ in LOS per year, which is unlikely to be clinically important. A calendar year random coefficient model was also fitted (online supplementary table S1) which suggested that changes in LOS through time varied significantly between centres, with no national pattern.

Finally, increased logistic EuroSCORE was associated with increased odds of prolonged LOS in surgeon only, anaesthetist only and cross-classified models (OR 0.784, $95 \%$ CI 0.768 to $0.800 ; 0.775,95 \%$ CI 0.759 to 0.791 and $0.785,95 \%$ CI 0.769 to 0.801 , respectively, $\mathrm{p}$ value $<0.0001$ for all). The percentage of the variation in prolonged LOS attributable to EuroSCORE (and remaining patient heterogeneity) was 96.05 (table 3 ). The variation attributable to the centre, surgeon and anaesthetist was quantified as $1.66 \%, 2.12 \%$ and $0.17 \%$, respectively.

We conducted exploratory analysis of the effect of age and logistic EuroSCORE on between-centre variation. Postoperative LOS increased by about $1 \%$ for an increase of 1 year in age (online supplementary table S2).
Although small, there was some variation between centres in the age effects, suggesting that part of the betweencentre variation could be ascribed to differences in the average age of the treated population. There was some variation between centres in the case-mix risk treated, which may explain part of the variation in centres' LOS (online supplementary table S3).

\section{DISCUSSION}

Our study cohort included 10 of 36 UK cardiothoracic surgical centres, totalling 107038 heterogeneous patients, equivalent to almost one third of the total cardiac operations performed in the UK during our study period. Patient risk factors accounted for over $95 \%$ of the variation in LOS and prolonged LOS in all models. The second most influential factor was the operating surgeon, with centre having a more moderate yet significant effect, whereas anaesthetist-induced variation was minimal.

\section{Comparison with other studies}

Our findings are consistent with published literature in other surgical fields suggesting much of the non-patient 
4A)

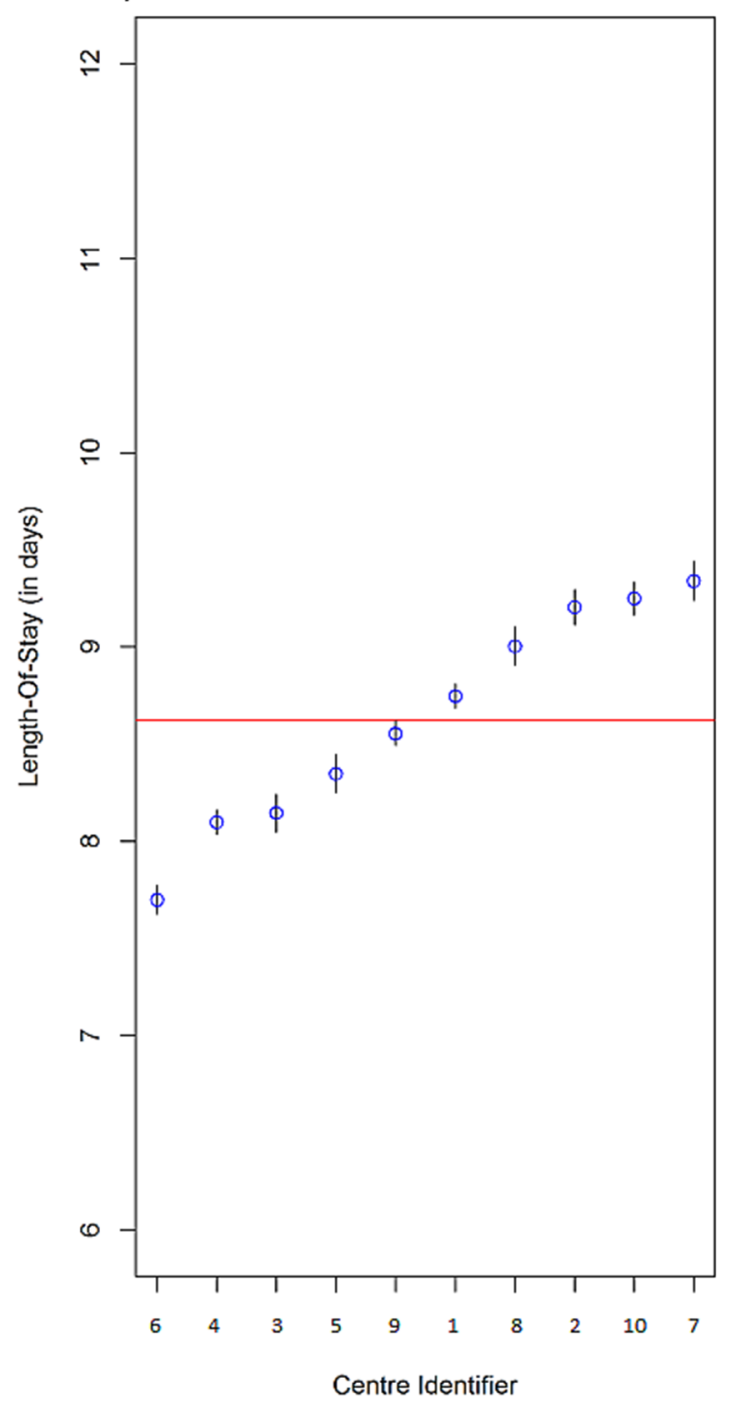

4B)

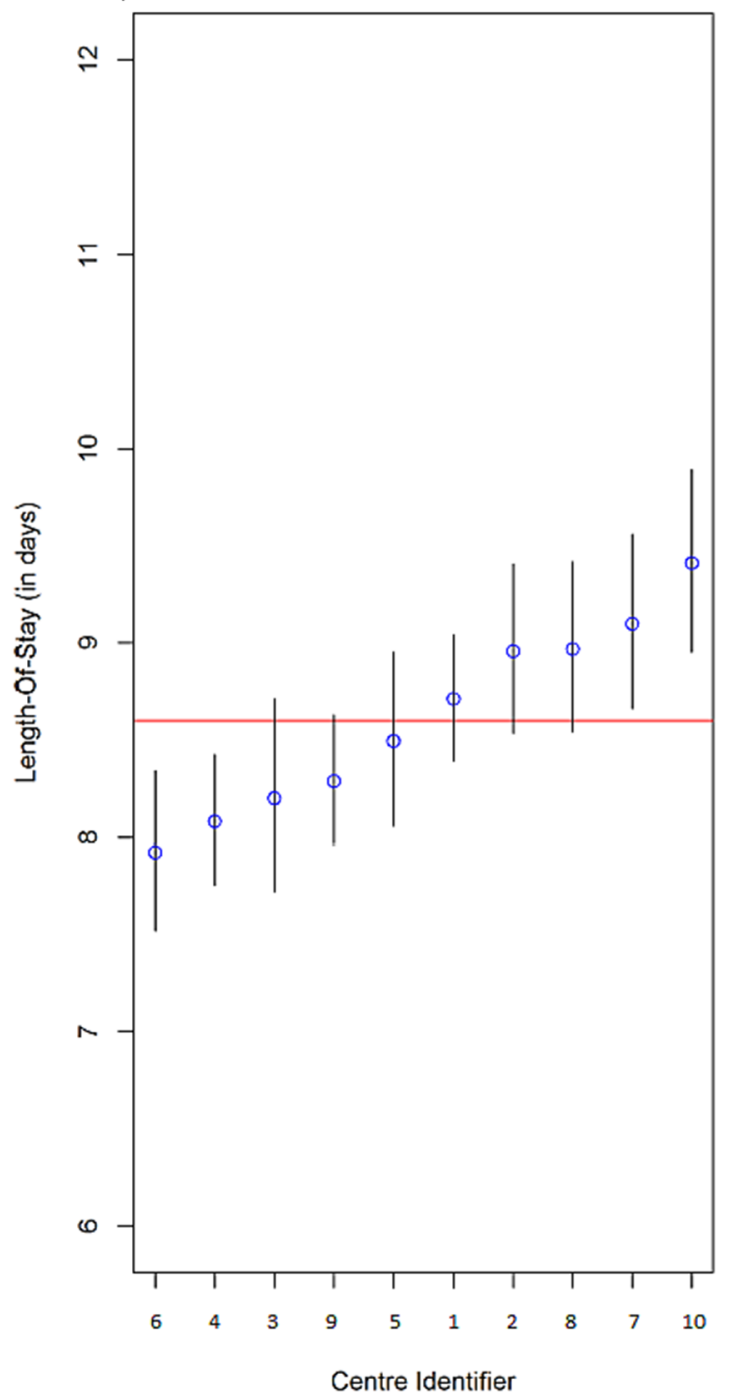

Figure 4 Estimated mean postoperative length of stay (LOS) in hospital and 95\% Cl for each centre (A, B) for a patient with average EuroSCORE risk. Horizontal line is the estimated average LOS for a patient with average EuroSCORE.

variation in LOS derives from different provider practices ${ }^{17}$ with the surgeon being a more influential component than the anaesthetist. This is to be expected as the surgeon (unlike the anaesthetist) has the oversight of the patients' postoperative ward care and discharge. In previously published work using this cohort, similar surgeon and anaesthetist effects were found for in-hospital mortality, with surgeons having a considerable impact $(4.00 \%)$ and anaesthetists a negligible effect $(0.25 \%) .{ }^{7}$ In contrast, there were no centre effects on in-hospital mortality. The centre importantly includes critical care and high dependency services, which may exert a significant effect on LOS, although it is difficult to isolate this aspect from other contributing factors using routinely collected data.

\section{Potential explanations and implications of findings}

Our findings suggest that differences in centre infrastructure, policies and possibly geographical location are more likely to affect postoperative LOS than patient survival. We conducted sensitivity analysis by re-estimating effects including cases of immediate discharge (i.e. zero LOS) and including all remaining cases that resulted in in-hospital death yielding very slightly reduced ICC estimates $(1.21 \%, 2.21 \%$ and $0.15 \%$ for centre, surgeon and anaesthetist, respectively). This reflects the fact that LOS for these patients is partly driven by mortality, resulting in reduced influence of external factors such the centre or surgeon. We further conducted exploratory analysis of factors that may contribute to increased between-centre variation. Our analysis supports the hypothesis that centres in areas with elderly populations are associated with increased LOS, in line with published evidence suggesting older patients are less likely to be discharged home (online supplementary table S2). ${ }^{14} 171829$ Likewise, exploratory analysis showed some between-centre variation in case-mix risk treated, which may explain part of the variation in centres' LOS (online supplementary table S3). 
The estimated mean LOS per surgeon (figure 3) appears superficially very similar to the estimated probability of in-hospital death per surgeon previously published (figure 2). ${ }^{7}$ We examined which surgeons were significantly below or above average both for in-hospital death and LOS, but there was no discernible pattern.

Figure 4 illustrates a relatively tight distribution of average LOS between centres. It is notable that the two centres (6 and 4) with shortest LOS, had the lowest average EuroSCOREs (4.29 additive and 5.77 logistic, respectively). In contrast, centre 10 may have been expected to have a shorter LOS given the relatively low average EuroSCORE (6.9). Geographical location may influence centres' LOS due to the type of populations treated. For instance, centres in less affluent areas, where access to home care is limited, may be associated with longer LOS. Alternatively, in areas with communities that have an established infrastructure and tradition of caring for relatives, centres may have shorter LOS. Further, in-depth examination of the association of location and socioeconomic status is needed in order to robustly estimate their impact on the LOS. The small decrease in LOS through time may result from improvements in the delivery of care in recent years, and is consistent with other published literature reporting longer hospitalisations at the beginning of the cohorts studied. ${ }^{21}$ Given the number of initiatives purporting to reduce LOS after cardiac surgery, the actual $1 \%$ per year reduction is modest. Although predicted LOS for a patient of average risk decreased over time in most centres, it increased in three (figure 5); this may be due to changes in management strategies, introduction of more conservative discharge practices in these centres or changes in patient-related factors. A potential risk of reducing LOS is an increased risk of hospital readmission due to premature hospital discharge. In April 2011, the Department of Health introduced a policy of non-payment for emergency readmissions to English hospitals. According to the 2011/2012 Payment by Results guidance, commissioners will no longer pay for any eligible emergency readmissions to a hospital within 30 days of discharge following planned hospital stay. The potential loss of considerable income may have induced reluctance of early postoperative discharge in some centres.

\section{Limitations}

1. Our study is limited by the lack of detailed patientrelated information, such as ethnic and social background, rural residency, availability of home carer, access to transportation and local resources

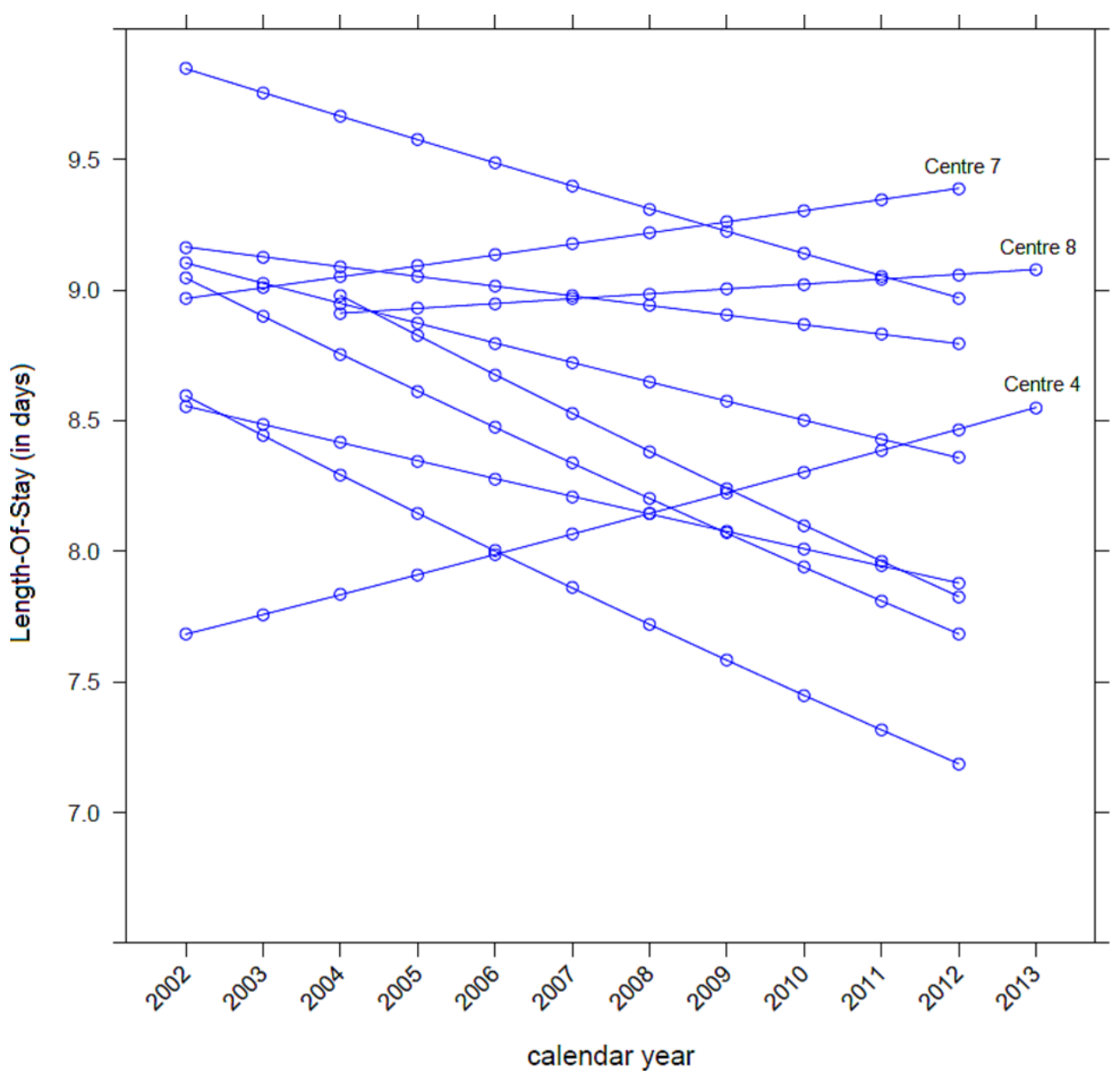

Figure 5 Predicted postoperative length of stay (LOS) in hospital, for a patient with average EuroSCORE risk, in each centre over time. 
for the provision of social services, which may have a significant effect on postoperative LOS.

2. We did not have access to centre characteristics, such as proportion of LOS spent in intensive care unit (ICU), high dependency unit (HDU) or postsurgical ward care. Different models of care, resulting in differing proportions of time in each ward type, could affect total LOS. Similarly, characteristics that may influence LOS, such as nurse-bed ratio, were not available. ${ }^{16} 18$

3. Information on other healthcare professionals involved in the patients' postoperative care that may contribute to variation in the LOS, such as ICU, HDU and ward staff was not available.

4. The logistic EuroSCORE is a predictive risk score for in-hospital mortality and may be less effective at capturing risk of increased LOS. The recalibrated EuroSCORE II, additionally including poor mobility (or frailty) as a risk factor, may be better at capturing risk of increased LOS.

5. Total hospital LOS may be underestimated due to lack of information on periods of time after inter-hospital transfer.

6. Our cohort included a relatively small number $(n=10)$ of high volume, specialist centres with a likely interest in quality improvements. Therefore, our results may not generalise to smaller, non-specialist centres and may be prone to underestimation of centre variation. Participating centres comprise a limited sample of all eligible centres and as such may also differ in average case mix or between-provider variability compared with non-participating centres. Nevertheless, as cardiac surgery in the UK is only offered in specialist cardiac centres with academic/teaching status, we would expect the participating and non-participating centres to be relatively similar in nature.

\section{Recommendations and future research}

Analysis of large Electronic Health Records can highlight characteristics of the centre and surgeon that introduce variation in patient outcomes. Future studies of more detailed databases examining features which may distinguish 'long' to 'short LOS' centres are required; potential key LOS drivers include varying discharge schemes, management strategies in preoperative/postoperative care, staffing levels, infrastructure and equipment available, such as operating theatres, medication and medical devices. ${ }^{16} 18{ }^{30}$ Likewise, further studies could identify provider practices and techniques that contribute to reduced LOS, such as level of accreditation, caseload volume and previous training and experience.

Delays in hospital discharge are mainly driven by postoperative patient-related complications and differences in centre and surgeon policies and practices; the NHS has previously highlighted that LOS is linked with differences in patient management. ${ }^{19}$ It is difficult to separate which result from an internal hospital culture, and which are the result of external local healthcare resources. We used sophisticated statistical methods to establish the degree to which postoperative LOS after cardiac surgery is affected by heterogeneity in patient risk, compared with other factors such as differences in centre policies and provider practice styles. Enhancing our understanding of the relationship between these patient-extraneous factors and postoperative LOS will help centres, providers and commissioners implement measures to enhance the efficiency of healthcare provision, minimise time in hospital and reduce excess resource use. Health systems, such as the NHS, can benefit considerably as, due to the high throughput, even small LOS reductions may result in large cost savings. ${ }^{16}$

\section{CONCLUSION}

We have shown that patient risk profile is the primary determinant of variation in LOS, thus current initiatives to reduce LOS by modifying consultant performance or local practice will have limited success. This implies that substantially reducing hospital stay requires shifting away from a one-size-fits-all approach to cardiac surgical care and investing in seeking alternative treatment options , personalised to high-risk patients.

\section{Author affiliations \\ ${ }^{1}$ Department of Medical Statistics, London School of Hygiene and Tropical Medicine, London, UK \\ ${ }^{2}$ Department of Anaesthesia and Intensive Care, Papworth Hospital, Cambridge, UK \\ ${ }^{3}$ Department of Cardiothoracic Surgery, Papworth Hospital, Cambridge, UK \\ ${ }^{4}$ Department of Anaesthesia and Intensive Care, St George's Hospital, London, UK}

Collaborators The contributors of ACTACC include G Lau (Glenfield Hospital, Leicester, UK); D Woodward (Northern General Hospital, Sheffield, UK); J Hillier (University Hospitals, Bristol, UK); M Ware (University Hospital of Wales, Cardiff, UK); S Agarwal (Liverpool Heart and Chest Hospital, Liverpool, UK); M Bill (Royal Victoria Hospital, Belfast, UK); R Gill (University Hospital, Southampton, UK); D Duthie (Leeds General Infirmary, Leeds, UK); H Skinner (City Hospital, Nottingham, UK).

Contributors OP, LDS and AAK conceived the study. OP and LDS designed the study. OP undertook the statistical analysis and drafted the manuscript. AAK and LDS contributed to the writing of the manuscript. AAK collected the data and coordinated the study. All authors interpreted the data, critically revised the manuscript and approved the final version. OP and LDS are joint guarantors for the study.

Funding The first author (OP) was partly funded by a Medical Research Council (MRC) PhD studentship and by a Gates Cambridge fellowship.

Competing interests OP declares partial funding by MRC and by a Gates Cambridge fellowship outside of the submitted work; AAK reports grants and personal fees from Pharmacosmos and personal fees from Vifor Pharma outside the submitted work.

Ethics approval The requirement for formal ethical approval was waived according to the National Research Ethics Service of the NHS Health Research Authority.

Provenance and peer review Not commissioned; externally peer reviewed. Data sharing statement № additional data available.

Open Access This is an Open Access article distributed in accordance with the Creative Commons Attribution Non Commercial (CC BY-NC 4.0) license, which permits others to distribute, remix, adapt, build upon this work non-commercially, and license their derivative works on different terms, provided the original work is properly cited and the use is non-commercial. See: http://creativecommons.org/ licenses/by-nc/4.0/ 
(c) Article author(s) (or their employer(s) unless otherwise stated in the text of the article) 2017. All rights reserved. No commercial use is permitted unless otherwise expressly granted.

\section{REFERENCES}

1. Blue Book Online. The Society for Cardiothoracic Surgery in Great Britain \& Ireland. 2017 http://bluebook.scts.org/\#ActivityRates (cited 14 March 2017).

2. Ducel G, Fabry J, Nicolle L. Prevention of hospital acquired infections: a practical guide, 2002.

3. Khan NA, Quan H, Bugar JM, et al. Association of postoperative complications with hospital costs and length of stay in a tertiary care center. J Gen Intern Med 2006;21:177-80.

4. Kaboli PJ, Go JT, Hockenberry J, et al. Associations between reduced hospital length of stay and 30-day readmission rate and mortality: 14-year experience in 129 Veterans Affairs hospitals. Ann Intern Med 2012;157:837-45.

5. Agency for Healthcare Research Quality. Interim Update on 2013 Annual Hospital-Acquired Condition Rate and Estimates of Cost Savings and Deaths Averted From 2010 to 2013. 2015 . (cited 2017 May 9) https://www.ahrq.gov/sites/default/files/wysiwyg/ professionals/quality-patient-safety/pfp/interimhacrate2013.pdf.

6. NCBC. National Cardiac Benchmarking Collaborative . 2017. cited 14 March 2017 http://www.ncbc-nhs.net/.

7. Papachristofi $\mathrm{O}$, Sharples LD, Mackay JH, et al. The contribution of the anaesthetist to risk-adjusted mortality after cardiac surgery. Anaesthesia 2016;71:138-46.

8. Papachristofi O, Mackay J, Powel S, et al. Impact of anesthetist and surgeon on cardiac surgical outcomes. JCVA 2014;28:103-9.

9. Birkmeyer JD, Finks JF, O'Reilly A et al. Surgical skill and complication rates after bariatric surgery. $N$ Engl $\mathrm{J}$ Med 2013;369:1434-42.

10. Hollenbeck BK, Roberts WW, Wolf JS. Importance of perioperative processes of care for length of hospital stay after laparoscopic surgery. J Endourol 2006;20:776-81.

11. Papachristofi $O$. Statistical issues in the design and analysis of randomised clinical trials of complex surgical interventions. University of Cambridge.2015.

12. Ramsay CR, Grant AM, Wallace SA, et al. Statistical assessment of the learning curves of health technologies. Health Technol Assess 2001:5:1-79.

13. De Hert SG, Van der Linden PJ, Cromheecke S, et al. Choice of primary anesthetic regimen can influence intensive care unit length of stay after coronary surgery with cardiopulmonary bypass. Anesthesiology 2004;101:9-20.

14. Hillis LD, Smith PK, Anderson JL, et al. ACCF/AHA guideline for coronary artery bypass graft surgery: a report of the American College of Cardiology Foundation/American Heart Association Task Force on Practice Guidelines. J Am Coll Cardiol 2011;58:e123-210.
15. van Mastrigt GA, Maessen JG, Heijmans J, et al. Does fast-track treatment lead to a decrease of intensive care unit and hospital length of stay in coronary artery bypass patients? A meta-regression of randomized clinical trials*. Crit Care Med 2006;34:1624-34.

16. Almashrafi $\mathrm{A}$, Alsabti $\mathrm{H}$, Mukaddirov $\mathrm{M}$, et al. Factors associated with prolonged length of stay following cardiac surgery in a major referral hospital in Oman: a retrospective observational study. BMJ Open 2016;6:e010764

17. Krell RW, Girotti ME, Dimick JB. Extended length of stay after surgery: complications, inefficient practice, or sick patients? JAMA Surg 2014;149:815-20.

18. Shinjo D, Fushimi K. Preoperative factors affecting cost and length of stay for isolated off-pump coronary artery bypass grafting: hierarchical linear model analysis. BMJ Open 2015;5:e008750.

19. NHS Institute for Innovation and Improvement. Length of Stay Reducing Length of Stay. http://www.institute.nhs.uk/quality_and_ service_improvement_tools/quality_and_service_improvement_tools/ length of stay.html (cited 14 March 2017).

20. Bucerius J, Gummert JF, Walther T, et al. Predictors of prolonged ICU stay after on-pump versus off-pump coronary artery bypass grafting. Intensive Care Med 2004;30:88-95.

21. Toumpoulis IK, Anagnostopoulos CE, Swistel DG, et al. Does EuroSCORE predict length of stay and specific postoperative complications after cardiac surgery? Eur J Cardiothorac Surg 2005;27:128-33.

22. Nashef SA, Roques F, Michel P, et al. European system for cardiac operative risk evaluation (EuroSCORE). Eur J Cardiothorac Surg 1999;16:9-13.

23. Michel P, Roques F, Nashef SA. Logistic or additive EuroSCORE for high-risk patients? Eur J Cardiothorac Surg 2003;23:684-7.

24. Zingone B, Pappalardo A, Dreas L. Logistic versus additive EuroSCORE. A comparative assessment of the two models in an independent population sample. Eur J Cardiothorac Surg 2004:26:1134-40.

25. Papachristofi O, Klein A, Sharples L. Evaluation of the effects of multiple providers in complex surgical interventions. Stat Med 2016;35:5222-46.

26. Rodriguez G, Elo I. Intra-class correlation in random-effects models for binary data. STATA Journal 2003;3:32-46.

27. Li B, Lingsma HF, Steyerberg EW, et al. Logistic random effects regression models: a comparison of statistical packages for binary and ordinal outcomes. BMC Med Res Methodol 2011:11:77-88.

28. Team RC. R: A Language and Environment for Statistical Computing. $R$ Foundation for Statistical Computing. Vienna, Austria: R Foundation for Statistical Computing, 2014.

29. Kurki TS, Häkkinen U, Lauharanta J, et al. Evaluation of the relationship between preoperative risk scores, postoperative and total length of stays and hospital costs in coronary bypass surgery. Eur J Cardiothorac Surg 2001;20:1183-7.

30. Widyastuti $Y$, Stenseth $R$, Wahba A, et al. Length of intensive care unit stay following cardiac surgery: is it impossible to find a universal prediction model? Interact Cardiovasc Thorac Surg 2012; 15:825-32. 